\title{
Monocyte/macrophage and $T$ cell activation markers are not independently associated with MI risk in healthy individuals - results from the HUNT Study
}

Thor Ueland, $\mathrm{PhD}^{1}$, Lars E. Laugsand, $\mathrm{MD}, \mathrm{PhD}^{2}$, Lars J. Vatten, $\mathrm{MD}, \mathrm{PhD}^{3}$, Imre Janszky, $\mathrm{MD}, \mathrm{PhD}^{4}$, Carl Platou, $\mathrm{MD}^{5}$, Annika E. Michelsen, $\mathrm{PhD}^{6}$, Jan K. Damås, MD, PhD ${ }^{7}$, Pål Aukrust, $\mathrm{MD}, \mathrm{PhD}^{8}$, Bjørn O. Åsvold, $\mathrm{MD}, \mathrm{PhD}^{9}$

${ }^{1}$ Research Institute of Internal Medicine, Oslo University Hospital Rikshospitalet; Faculty of Medicine, University of Oslo, Oslo; K.G. Jebsen TREC, University of Troms $\varnothing$, Troms $\varnothing$, Norway. This author takes responsibility for all aspects of the reliability and freedom from bias of the data presented and their discussed interpretation

${ }^{2}$ Department of Public Health and Nursing, NTNU, Norwegian University of Science and Technology; Department of Cardiology, St. Olavs Hospital, Trondheim, Norway. This author takes responsibility for all aspects of the reliability and freedom from bias of the data presented and their discussed interpretation.

${ }^{3}$ Department of Public Health and Nursing, NTNU, Norwegian University of Science and Technology, Trondheim, Norway. This author takes responsibility for all aspects of the reliability and freedom from bias of the data presented and their discussed interpretation.

${ }^{4}$ Department of Public Health and Nursing, NTNU, Norwegian University of Science and Technology, Trondheim, Norway; Department of Public Health Sciences, Karolinska Institutet, Stockholm, Sweden. This author takes responsibility for all aspects of the reliability and freedom from bias of the data presented and their discussed interpretation.

${ }^{5}$ Department of Internal Medicine, Levanger Hospital, Nord-Trøndelag Hospital Trust, Levanger, Norway. This author takes responsibility for all aspects of the reliability and freedom from bias of the data presented and their discussed interpretation.

${ }^{6}$ Research Institute of Internal Medicine, Oslo University Hospital Rikshospitalet, Oslo, Norway. This author takes responsibility for all aspects of the reliability and freedom from bias of the data presented and their discussed interpretation.

${ }^{7}$ Centre of Molecular Inflammation Research, Department of Cancer Research and Molecular Medicine, NTNU, Norwegian University of Science and Technology; Department of Infectious Diseases, St. Olavs Hospital, Trondheim, Norway. This author takes responsibility for all aspects of the reliability and freedom from bias of the data presented and their discussed interpretation.

${ }^{8}$ Research Institute of Internal Medicine, ${ }^{2}$ Section of Clinical Immunology and Infectious Diseases Oslo University Hospital Rikshospitalet; ${ }^{3}$ Faculty of Medicine, University of Oslo, Oslo; K.G. Jebsen TREC, University of Troms $\varnothing$, Troms $\varnothing$, Norway. This author takes 
responsibility for all aspects of the reliability and freedom from bias of the data presented and their discussed interpretation.

${ }^{9}$ Department of Public Health, NTNU, Norwegian University of Science and Technology; Department of Endocrinology, St. Olavs Hospital, Trondheim, Norway. This author takes responsibility for all aspects of the reliability and freedom from bias of the data presented and their discussed interpretation.

Corresponding Author: Thor Ueland, Research Institute of Internal Medicine, Oslo University Hospital, Rikshospitalet, P.B. 4950 Nydalen, 0424 Oslo, Norway. Phone: 47-23073626, e-mail: thor.ueland@medisin.uio.no

Acknowledgement of grant support: NTNU (L.E.L, I.J., C.P.), Central Norway Regional Health Authority (L.E.L, I.J.), Swedish Council of Working Life and Social Research and Swedish Research Council (I.J).

Conflict of Interest: none declared

Key words: leukocyte markers, myocardial infarction 


\section{ABSTRACT}

Background: We hypothesized that circulating markers reflecting monocyte/macrophage and T cell activation are associated with increased risk of myocardial infarction (MI) in apparently healthy individuals.

Methods: Serum monocyte/macrophage and T cell activation markers soluble (s) CD163, sCD14, Gal3BP, sCD25 and sCD166 were analyzed by enzyme-immunoassay in a casecontrol study nested within the population-based HUNT2 cohort in Norway. Among 58,761 apparently healthy men and women followed a median 11.3 years, 1,587 incident MI cases were registered, and compared to 3,959 age- and sex-matched controls.

Results: Higher serum sCD163 (Q4 vs. Q1 OR: 1.27, P-trend 0.002), sCD14 (Q4 vs. Q1 OR: 1.38, $P$-trend $<0.001)$, and especially sCD25 (Q4 vs. Q1 OR: 1.45, $P$-trend $<0.001)$, were associated with increased MI risk in the age-and sex adjusted models. However, after additional adjustment for cardiovascular risk factors these associations were strongly attenuated (Q4 vs Q1 ORs between 1.02-1.12, $P$-trends between $0.30-0.58)$.

Conclusions: sCD163, sCD14 and sCD25 may reflect leukocyte activation and inflammatory mechanisms related to atherogenesis, but do not predict MI risk above and beyond conventional cardiovascular risk factors. 


\section{Introduction}

Inflammation may play a central role at all stages of atherogenesis, and activation of inflammatory cells including monocytes/macrophages and $\mathrm{T}$ cells occur from the earliest stage of atherosclerosis but the cytokines and chemokines released from these cells often circulate at relatively low level which limits their utility as clinical markers (1). We hypothesized that circulating levels of stable and readily detectable markers of leukocyte activation (2-4), reflecting both monocyte/macrophage (soluble [s] CD163 (5), sCD14 (6), galectin 3 binding protein [Ga13BP] (7) and/or T-cell (sCD25/soluble interleukin 2 receptor [sIL-2R](8), sCD166/ALCAM](9)) could reflect early activation of these inflammatory cells in apparently heathy individuals who later experienced an incident MI. These markers represent relatively specific surface molecules that are cleaved (sCD163, sCD14 and sCD25) or released (Gal3BP, sCD166) from their respective cells upon activation under inflammatory conditions and increased levels have been reported in patients with established cardiovascular disease (CVD) (10-14). Therefore, we investigated the associations between circulating levels of these markers and risk of MI in a nested case-control study within a large population-based cohort in Norway (the HUNT Study).

\section{Patients and Methods}

We conducted a case-control study nested within the second survey of the HUNT Study. All residents in Nord-Trøndelag aged 20 years or older were invited to attend between August 1995 and June 1997 as described in detail elsewhere (15). Among 93,898 invited individuals, 65,215 attended a clinical examination and of these 5,221 were excluded because they reported a history of MI, angina pectoris or stroke, and 1,233 participants were excluded due to missing values on relevant covariates, leaving 58,761 participants eligible for follow-up. Among individuals with stored serum, 1,587 participants were diagnosed in hospital with a first incident MI according to the European Society of Cardiology/American College of Cardiology consensus guidelines (16), during a mean follow-up of 11.3 years and 3,959 participants were matched to the cases by age at risk and by sex using incidence density sampling, the recommended procedure for nested case-control studies (17). Clinical information at baseline was collected as described elsewhere (15). The study protocol conforms to the 
ethical guidelines of the 1975 Declaration of Helsinki. The study was approved by the regional committee for ethics in medical research, by the National Directorate of Health, and by the Norwegian Data Inspectorate. Written informed consent to participate in the study was obtained from each participant.

\section{Serum leukocyte markers and high-sensitivity (hs) C-reactive protein (CRP)}

Peripheral venous blood was drawn into pyrogen-free tubes without any additives. After clotting at room temperature, the tubes were centrifuged at $1,500 \mathrm{~g}$ for 10 minutes, and serum was stored at $70^{\circ} \mathrm{C}$. Serum levels of sCD14 (product no. DY383/intra-assay CV: 2.2\% /inter-assay CV: $7.3 \%$ ), sCD163 (DY1607/2.9\%/3.9\%), sCD25 (DY223/3.1\%/8.9\%), sCD166 (DY656/3.6\%/6.4\%), Gal3BP (DY2226/6.5\%/9.5\%) and hsCRP (DY1707/3.0\%/9.1\%) were measured in duplicate by enzyme immune-assay with matched antibodies obtained from R\&D Systems (Minneapolis, MN) in a 384 format using the combination of a SELMA (Jena, Germany) pipetting robot and a BioTek (Winooski, VT, USA) dispenser/washer. Absorption was read at $450 \mathrm{~nm}$ with wavelength correction set to $540 \mathrm{~nm}$ using an ELISA plate reader (Bio-Rad, Hercules, CA, USA)

\section{Statistical analysis}

Pearson correlation coefficients were calculated to evaluate correlation between leukocyte markers and the other covariates. We used conditional logistic regression analysis to estimate age- and sexadjusted odds ratios (ORs, with 95\% confidence intervals [CIs]) of MI according to quartile categories of leukocyte marker concentrations, based on the quartile distribution of these markers among the controls. As statistical analyses were stratified on groups of each case and his/ her matched controls using conditional logistic regression analysis, the unequal number of controls for each case did not bias the estimates. For tests of trend of the association across leukocyte marker quartiles, we assigned each participant the median leukocyte marker level within his/her quartile, and treated these median values as a continuous variable in the logistic models. Additionally, we estimated MI risk according to leukocyte marker levels expressed by restricted cubic splines (with 5 knots) to assess linearity of the associations of leukocyte markers with MI risk. 
In the multivariable analyses of leukocyte marker levels and MI risk, we adjusted for the following variables: BMI, total cholesterol, HDL cholesterol, systolic blood pressure, diastolic blood pressure, and log transformed hsCRP and creatinine (continuous variables) as well as smoking (current, previous, or never smoking) and diabetes mellitus (yes/no). In a sensitivity analysis, we analyzed the first 5 years and the rest of the follow-up separately to assess whether the associations of leukocyte markers and risk of early MI events differed from risk of late MI events. All statistical analyses were conducted in STATA version 12.2 for Windows (StataCorp LP, College Station, LP).

\section{Results}

A total of 1,587 participants who participated in the HUNT2 study were diagnosed at hospital with a first incident MI during a mean follow-up of 11.3 years. Baseline levels $(\mathrm{ng} / \mathrm{mL})$ of the leukocyte markers in cases $(n=1,587)$ and controls $(n=3,959)$, respectively, where: $\mathrm{sCD} 14,2.9 \pm 1.0$ vs. $2.8 \pm 1.0$; sCD163, $201 \pm 127$ vs. $195 \pm 118$; Gal3BP, $5.9 \pm 2.9$ vs. $5.8 \pm 2.8$; sCD66 $62.0 \pm 16.4$ vs. $62.4 \pm 16.2$; sCD25, $0.66 \pm 0.31$ vs $0.63 \pm 0.29$. Baseline characteristics of study population are shown in Table 1 . Serum leukocyte markers correlated moderately with other cardiovascular risk markers, in particular hsCRP $(\mathrm{r}=0.19-0.28, p<0.001)$ (Table 1$)$.

We expressed serum leukocyte marker concentrations both as quartile $(\mathrm{Q})$ categories and as continuous variables (expressed as restricted cubic splines). sCD163, sCD14, and especially sCD25, were positively associated with MI risk in the age-and sex adjusted model (Figure 1). However, after additional adjustment for cardiovascular risk factors these associations were strongly attenuated (Figure 1). There was no association of serum Gal3BP and sCD166 with risk of MI. Sensitivity analysis revealed similar associations of leukocyte markers and risk for early and late MI events.

\section{Discussion}

Recruitment and activation of inflammatory cells including monocytes/macrophages and $\mathrm{T}$ cells is considered one of the earliest cellular responses in atherosclerotic lesion formation although the majority of research is derived from experimental models (1). Our finding in this large prospective population study of individuals free of clinical CVD at baseline, that serum levels of sCD163, sCD14, 
and SCD25 were related to increased risk of MI supports a role for leukocyte activation in the progression of clinical atherosclerosis.

Upon activation under pro-inflammatory conditions, monocytes and tissue macrophages may release sCD163 (18) and sCD14 (6) into the circulation and increased levels have been demonstrated in patients with coronary artery disease (CAD) $(10,14)$. Moreover, for SCD163, a positive association with carotid intima media thickness was shown in individuals free from clinical CVD (19), and enhanced expression was seen in unstable $v s$. stable plaques (20). Conversely, a small nested casecontrol study reported no significant association between sCD14 and risk of MI (13). A truncated soluble form of the IL-2 receptor (i.e. sCD25) is secreted from activated T cells upon stimulation with IL.2. Elevated sCD25 is observed in patients with CAD (12), but there are no data on risk of MI in individuals free of clinically diagnosed CVD.

The present study is by far the largest study to investigate the leukocyte markers sCD14, sCD163, Gal3BP, sCD166 and sCD25 in relation to risk of MI during follow-up in apparently healthy individuals. These markers correlated moderately with several CVD risk factors and hsCRP in particular. However, after adjustment for established CVD risk factors, the strength of the association with MI risk was strongly attenuated. The present work has some important limitations. Similar to other studies, the assays were not standardized making comparisons of levels with other studies difficult. Moreover, the samples were stored for 11-13 years which could influence the association with MI risk. However, all samples had equal volumes and freeze/thaw cycles, and thus it is unlikely any storage effects would have influenced cases and controls differently. Finally, presence of overt inflammatory/infectious disease was not assessed at baseline and therefore we cannot $t$ exclude that mild infection or inflammatory disorders among the participants could have influenced our results.

Our results suggest that sCD163, sCD14 and sCD25 may reflect leukocyte activation and inflammatory mechanisms related to atherogenesis, but they do not predict MI risk above and beyond conventional cardiovascular risk factors.

Acknowledgements: We thank the Department for Research and clinicians at the Medical Department, Nord-Trøndelag Hospital Trust, Norway, for extracting the data from the patient records. 
The HUNT Study is collaboration between HUNT Research Centre (Faculty of Medicine, Norwegian

University of Science and Technology [NTNU]), Nord-Trøndelag County Council, Central Norway

Health Authority, and the Norwegian Institute of Public Health.

\section{References}

1. Hansson GK, Jonasson L. The discovery of cellular immunity in the atherosclerotic plaque. Arteriosclerosis, thrombosis, and vascular biology 2009;29:1714-7.

2. Wang J, Zhu HH, Xue JH, Wu SS, Chen Z. Effects of storage conditions on the stability of serum CD163, NGAL, HMGB1 and MIP2. International journal of clinical and experimental pathology 2015;8:4099-105.

3. Aziz N, Nishanian P, Mitsuyasu R, Detels R, Fahey JL. Variables that affect assays for plasma cytokines and soluble activation markers. Clinical and diagnostic laboratory immunology 1999;6:89-95.

4. Kulasingam V, Zheng $\mathrm{Y}$, Soosaipillai A, Leon AE, Gion M, Diamandis EP. Activated leukocyte cell adhesion molecule: a novel biomarker for breast cancer. International journal of cancer 2009;125:9-14.

5. Davis BH, Zarev PV. Human monocyte CD163 expression inversely correlates with soluble CD163 plasma levels. Cytometry Part B, Clinical cytometry 2005;63:16-22.

6. Shive $\mathrm{CL}$, Jiang $\mathrm{W}$, Anthony DD, Lederman MM. Soluble CD14 is a nonspecific marker of monocyte activation. Aids 2015;29:1263-5.

7. Shaked I, Hanna DB, Gleissner $\mathrm{C}$ et al. Macrophage inflammatory markers are associated with subclinical carotid artery disease in women with human immunodeficiency virus or hepatitis C virus infection. Arteriosclerosis, thrombosis, and vascular biology 2014;34:1085-92.

8. Rubin LA, Kurman CC, Fritz ME et al. Soluble interleukin 2 receptors are released from activated human lymphoid cells in vitro. Journal of immunology 1985;135:3172-7.

9. Bowen MA, Patel DD, Li $X$ et al. Cloning, mapping, and characterization of activated leukocyte-cell adhesion molecule (ALCAM), a CD6 ligand. The Journal of experimental medicine 1995;181:2213-20.

10. Aristoteli LP, Moller HJ, Bailey B, Moestrup SK, Kritharides L. The monocytic lineage specific soluble CD163 is a plasma marker of coronary atherosclerosis. Atherosclerosis 2006;184:3427.

11. Gleissner CA, Erbel C, Linden F et al. Galectin-3 binding protein plasma levels are associated with long-term mortality in coronary artery disease independent of plaque morphology. Atherosclerosis 2016;251:94-100.

12. Sakamoto A, Ishizaka N, Saito K et al. Serum levels of IgG4 and soluble interleukin-2 receptor in patients with coronary artery disease. Clinica chimica acta; international journal of clinical chemistry 2012;413:577-81.

13. Smedbakken L, Jensen JK, Hallen J et al. Activated leukocyte cell adhesion molecule and prognosis in acute ischemic stroke. Stroke; a journal of cerebral circulation 2011;42:2453-8.

14. Zalai CV, Kolodziejczyk MD, Pilarski L et al. Increased circulating monocyte activation in patients with unstable coronary syndromes. J Am Coll Cardiol 2001;38:1340-7.

15. Laugsand LE, Vatten L, Platou C, Janszky I. Insomnia and the risk of acute myocardial infarction: a population study. Circulation 2011;124:2073-81.

16. Alpert JS, Thygesen K, Antman E, Bassand JP. Myocardial infarction redefined--a consensus document of The Joint European Society of Cardiology/American College of Cardiology Committee for the redefinition of myocardial infarction. J Am Coll Cardiol 2000;36:959-69. 
17. Rothman KJ, Greenland S, Lash TL. Modern Epidemiology. Third ed: Lippincott Williams \& Willkins, 2008.

18. Hintz KA, Rassias AJ, Wardwell $\mathrm{K}$ et al. Endotoxin induces rapid metalloproteinase-mediated shedding followed by up-regulation of the monocyte hemoglobin scavenger receptor CD163. Journal of leukocyte biology 2002;72:711-7.

19. Moreno JA, Munoz-Garcia B, Martin-Ventura JL et al. The CD163-expressing macrophages recognize and internalize TWEAK: potential consequences in atherosclerosis. Atherosclerosis 2009;207:103-10.

20. Yunoki K, Naruko T, Komatsu R et al. Enhanced expression of haemoglobin scavenger receptor in accumulated macrophages of culprit lesions in acute coronary syndromes. European heart journal 2009;30:1844-52. 


\section{Figure legend}

Figure 1. Associations between serum leukocyte marker concentrations and risk of incident MI.

Graphs show ORs (with 95\% CI) for MI by concentrations of leukocyte markers (expressed by restricted cubic splines), displayed from 2.5 to 97.5 percentiles of leukocyte markers (ng/mL except $\mathrm{sCD} 25: \mathrm{pg} / \mathrm{mL}$ ), based on the quantile distribution among controls with $5^{\text {th }}$ percentile as reference. Tables show ORs and 95\% CI for MI risk according to quartiles (Q) of the markers with Q1 as reference. Range for quartiles of markers in Supplementary Table 2. $P$ value: linear trend across categories 
Table 1. Baseline characteristics of the study population

\begin{tabular}{|c|c|c|c|c|c|c|c|}
\hline & \multirow{2}{*}{$\begin{array}{c}\text { Cases } \\
(\mathbf{n}=\mathbf{1 , 5 8 7})\end{array}$} & \multirow{2}{*}{$\begin{array}{c}\text { Controls } \\
(n=3,939)\end{array}$} & \multicolumn{5}{|c|}{ Correlation coefficents } \\
\hline & & & sCD14 & sCD163 & Gal3BP & sCD166 & sCD25 \\
\hline Male sex, $N(\%)$ & $981(61.8)$ & $2,532(64.0)$ & $-0.17 *$ & $-0.09 *$ & $-0.15^{*}$ & $-0.33 *$ & -0.02 \\
\hline Age at event, years & $70.2 \pm 12.5$ & $73.5 \pm 13.7$ & $0.13 *$ & $0.21 *$ & $0.12 *$ & $0.22 *$ & $0.18 *$ \\
\hline Diabetes mellitus, N (\%) & $115(7.3)$ & $204(5.2)$ & $0.11 *$ & $0.07 *$ & 0.04 & $-0.07 *$ & -0.01 \\
\hline Total cholesterol, mmol/L & $6.7 \pm 1.2$ & $6.3 \pm 1.2$ & $0.06^{*}$ & 0.04 & $0.06^{*}$ & $0.17 *$ & -0.04 \\
\hline HDL cholesterol, $\mathrm{mmol} / \mathrm{L}$ & $1.25 \pm 0.37$ & $1.37 \pm 0.41$ & $0.05 *$ & $-0.12 *$ & $-0.11 *$ & $0.05 *$ & $-0.10 *$ \\
\hline hsCRP, mg/L & $2.4(2.6)$ & $2.0(2.3)$ & $0.19 *$ & $0.28 *$ & $0.16^{*}$ & $0.20 *$ & $0.23 *$ \\
\hline Creatinine, $\mu \mathrm{mol} / \mathrm{L}$ & $91(19)$ & $90(18)$ & 0.02 & 0.03 & 0.00 & 0.00 & $0.18 *$ \\
\hline Triglycerides, mmol/L & $2.3 \pm 1.3$ & $1.9 \pm 1.2$ & -0.04 & $0.06^{*}$ & $0.16^{*}$ & 0.01 & 0.00 \\
\hline Body mass index, $\mathrm{kg} / \mathrm{m}^{2}$ & $27.4 \pm 4.0$ & $26.8 \pm 3.9$ & -0.02 & $0.21 *$ & $0.25 *$ & $0.06^{*}$ & 0.02 \\
\hline Systolic blood pressure, $\mathrm{mmHg}$ & $153 \pm 24$ & $150 \pm 24$ & $0.10 *$ & $0.18 *$ & $0.13 *$ & $0.12 *$ & $0.09 *$ \\
\hline Diastolic blood pressure, $\mathrm{mmHg}$ & $87 \pm 12$ & $85 \pm 12$ & $0.05 *$ & $0.09 *$ & $0.10^{*}$ & 0.01 & 0.03 \\
\hline Smoking, $\%$ (never/former/current) & $33.8 / 28.8 / 37.4$ & $41.3 / 34.4 / 24.3$ & 0.01 & $-0.11 *$ & -0.02 & $-0.13 *$ & $0.05^{*}$ \\
\hline
\end{tabular}


Figure 1. Associations between serum leukocyte marker concentrations and risk of incident MI
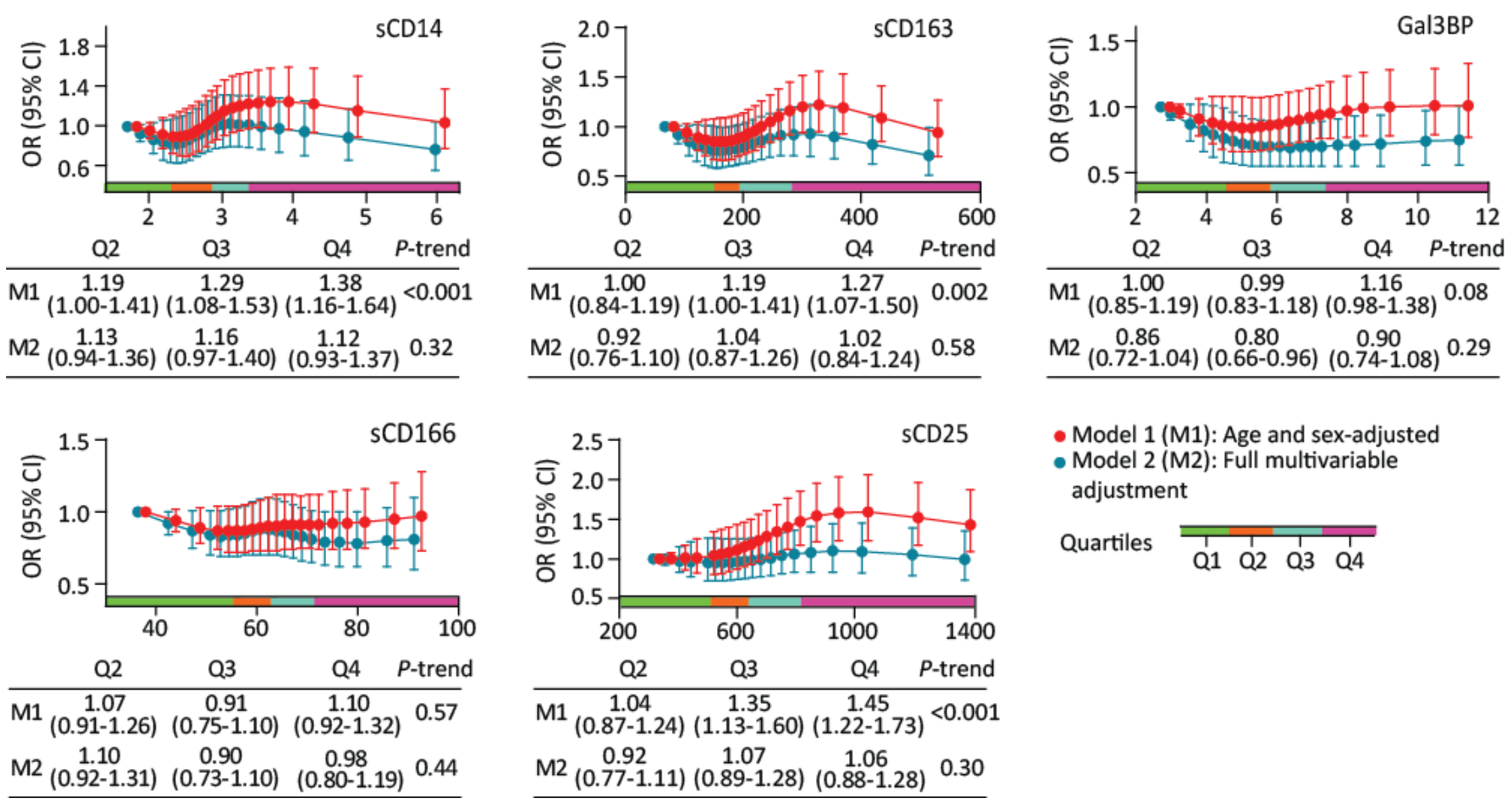

- Model 1 (M1): Age and sex-adjusted

- Model 2 (M2): Full multivariable

adjustment

Quartiles

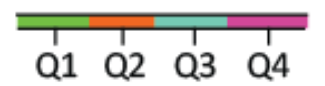

\begin{tabular}{|c|c|c|c|}
\hline Q2 & Q3 & Q4 & tive \\
\hline $\mathrm{M} 1_{(0.91-1.26)}^{1.07}$ & $\begin{array}{c}0.91 \\
(0.75-1.10)\end{array}$ & $\begin{array}{c}1.10 \\
(0.92-1.32)\end{array}$ & 0.5 \\
\hline M2 $\frac{1.10}{(0.92-1.31)}$ & $\begin{array}{c}0.90 \\
(0.73-1.10)\end{array}$ & $\begin{array}{c}0.98 \\
(0.80-1.19)\end{array}$ & ) \\
\hline
\end{tabular}

Graphs show ORs (with 95\% CI) for MI by concentrations of leukocyte markers (expressed by restricted cubic splines), displayed from 2.5 to 97.5

percentiles of leukocyte markers (ng/mL except sCD25: $\mathrm{pg} / \mathrm{mL}$ ), based on the quantile distribution among controls with $5^{\text {th }}$ percentile as reference. Tables show ORs and 95\% CI for MI risk according to quartiles (Q) of the markers with Q1 as reference. Range for quartiles of markers in Supplementary Table 2.

$P$ value: linear trend across categories. 